\title{
Pulse Combustion: The Quantification of Characteristic Times
}

\author{
J. O. KELLER and T. T. BRAMLETTE \\ Combustion Research Facility, Sandia National Laboratories, Livermore, CA 94551 \\ C. K. WESTBROOK \\ Lawrence Livermore National Laboratory, Livermore, CA 94551
}

and

J. E. DEC

Department of Mechanical Engineering, University of Michigan, Ann Arbor, MI

\begin{abstract}
Measurements of the total ignition delay time in a pulse combustor have been made for several chemical kinetic ignition delay times and several fluid dynamic mixing times. These measured total ignition delay times are compared with calculated values of the characteristic time for mixing and with calculated values for the homogeneous ignition delay time. A chemical kinetic model was used to calculate the homogeneous chemical kinetic ignition delay time for conditions typical of an operating pulse combustor. Similarly, a fluid dynamic mixing model was used to estimate characteristic times for a transient jet of cold reactants to mix with an ambient environment of hot products to an ignition temperature. These calculated time scales compared well with measured values in both trend and magnitude. It has also been shown that a simple sum of the characteristic mixing times and chemical kinetics times provides a good first-order approximation to the total ignition delay time.
\end{abstract}

\section{INTRODUCTION}

Most previous research on pulse combustors has been directed toward examinations of the overall characteristics such as heat transfer, efficiency, frequency of operation, and pollutant formation. Previous work has done little to elucidate the fundamental controlling physics of the pulse combustion process. Fundamental questions such as how to predict a priori whether a given system will resonate still remain unanswered [1].

Through testing a variety of configurations that had different "mixing" characteristics, it has long been known that fluid dynamic mixing is important to the operation of pulse combustors. However, the precise role of mixing has never been quantified experimentally or theoretically. Keller et al. [2] established the concept of individual characteristic delay times for pulse combustors (for example, time required to mix fuel and oxidizer, time required to mix the reactants with the hot products, time required for chemical kinetics, and the natural resonance time) and the idea that these times contribute nearly independently to a total ignition delay time. This work found that it is only their contributions to the total delay time that affected the phase relationship between the resonant pressure wave and the instantaneous energy release rate. This total delay time is one of the most important factors in determining the resonant response of the pulse combustor.

Although Keller et al. established the combustor's response to relative changes in these characteristic times, the relative and absolute magnitudes 
of these times remained unknown. The quantification of the individual characteristic times and their contributions to the total ignition delay time must be determined before widespread application of this information can be realized. The ability to predict the total ignition delay time and control its value provides the first step in moving the design of pulse combustion devices from trial and error to good engineering practices.

The physical and chemical processes leading to ignition are highly coupled temporally and spatially varying processes that involve turbulent mixing, injection rates, chemical kinetics, and so on. Current models of this combustion process - for example, two flame fronts each propagating in opposite directions, or an arbitrarily defined energy release rate as a function of time (e.g., a half sine wave)-are not very satisfying. Both of these approaches have been used in pulse combustion models with only modest success. The present work establishes some simple yet powerful techniques to quantify the effects on the total ignition delay time due to changes in the fluid dynamic mixing time and in the homogeneous chemical kinetic ignition delay time. It is recognized that these important processes overlap to some extent in a nonlinear fashion. Nevertheless, a significant amount of useful information can be extracted from the experimental results by treating the influence of these processes as independent.

In this paper we present a description of the numerical models used to calculate two of the characteristic times, first for the fluid dynamic mixing time and then for the homogeneous chemical kinetic time. The experimental facility and diagnostics used for model verification are described. The magnitude of the characteristic times that contribute to the total ignition delay time is discussed, as well as the general nature of these times and how they affect the performance of the pulse combustor.

\section{THEORETICAL MODELS AND EXPERIMENTAL FACILITY}

\section{Fluid Dynamic Mixing Model}

The mixing model of Rife and Heywood [3] was extended and applied recently to the injection pro- cess that occurs in a pulse combustor [4]. Their model is based on the classical steady two-phase turbulent jet model of Abramovich [5]. The model assumes similarity profiles for the radial distributions of velocity and concentration. By requiring conservation of mass and momentum, expressions are developed for the axial growth of the jet and the decay of centerline velocity and concentration. Rife and Heywood examined the rate at which a nonreacting, impulsively started, transient jet mixed to a certain equivalence ratio. Assuming that the turbulent Lewis number for the flow in the pulse combustor is 1 and that the turbulent Sherwood number in the impulsively started jet of Rife and Heywood is also 1, the same analysis can be used to calculate the rate at which the premixed reactants mix with hot combustion products from the previous pulse combustor cycle to a given temperature.

Bramlette [4] hypothesized that for the case of "fast" chemical kinetics (when the chemical ignition delay time scale is much less than the fluid dynamic mixing time scales of the combustor, $\tau_{\text {kinetic }} \ll \tau_{\text {mixing }}$ ) the rate of fluid dynamic mixing can be used to predict the timing and instantaneous rate of energy release in a pulse combustor. The injection process was modeled in an integral sense as an impulsively started jet with the same total mass and momentum flux as the temporally varying jet characteristic of a pulse combustor. It was shown that if the theoretical quasi-steady penetration time of the transient jet were modified by a single multiplicative factor, $C_{\text {pent }}$, then the theoretical predictions of penetration rate agreed well with a variety of experimental data for nonreacting, impulsively started jets with different injection velocities, initial radii, and density ratios.

The model was used to calculate the rate at which a transient jet of premixed fuel and air at temperature $T_{\mathrm{r}}$ achieved a temperature $T_{\mathrm{mix}}\left(T_{\mathrm{mix}}\right.$ was chosen to satisfy the "fast" kinetics assumption) when injected into combustion products at temperature $T_{\mathrm{p}}$. Calculations were performed to determine the sensitivity of the predicted mixing rate to mass flow rate, jet radius, combustion product temperature, density ratio, and the temperature $T_{\mathrm{mix}}$. All of these parameters were found to result in variations in mixing characteristics that 
would affect the performance of a pulse combustor, with mean mass flow rate producing the most dramatic effect. Thus, for the present work the mixing characteristics were altered by varying the mean mass flow rate.

This model as applied to the pulse combustor calculates the time history of many discrete elements of reactants. The integrated results from this Lagrangian calculation are used to provide a meaningful characteristic mixing time $\left(\tau_{\text {mixing }}\right.$ ) for the combustor as a whole.

There are several potential sources of error that may result from applying a quasi-steady mixing model to pulse combustors. First, such an approach neglects the influence of bulk fluid motion of the combustion products from the previous cycle. Based on earlier work [6] with an injection system more complex than the one used in the present study, it can be assumed that this effect is small. Second, the effect of combustion on the injection process and subsequent mixing is neglected. Because of the dependence of the mixing rate on density ratio, this approximation will lead to an underprediction of mixing rate [4] (and thus the instantaneous rate of energy release) during the latter part of the pulse combustion cycle. As a result, this model will tend to overpredict the duration of the high energy release rate. Finally, there is evidence [7-9] that the entrainment rate of a transient jet depends on both the jet centerline injection velocity and its time derivative. Hence, the use of a quasi-steady assumption in determining the value of $C_{\text {pent }}$ will cause an underprediction of the mixing rate. Thus, the value of $C_{\text {pent }}$ was determined so that the predicted mixing rate agreed with experimental data at one mass flow rate for the current pulse combustor configuration.

\section{Chemical Kinetics Model}

Calculations were carried out to estimate the characteristic chemical kinetic time scales in the pulse combustor. The model follows the time evolution of a given sample of gas that consists initially of a mixture of fuel, air, and residual products from previous cycles. The computations use the HCT code [10] with a detailed reaction mechanism [11-13] that has been thoroughly tested for the fuel and conditions of this study. The rate constants used in the current modeling analysis are tabulated in Ref. 13; however, that reaction mechanism includes chemical species as large as butane $\left(\mathrm{C}_{4} \mathrm{H}_{10}\right)$, so all of the hydrocarbon species with more than two $\mathrm{C}$ atoms and the elementary reactions involving them have been eliminated, leaving a $\mathrm{C}_{1}-\mathrm{C}_{2}$ mechanism for the oxidation of methane. The pressure during ignition is assumed to be constant at $101.3 \mathrm{kPa}$, spatial variations in temperature and species concentrations are neglected, and energy losses during ignition are not considered. These assumptions are reasonable as long as the homogeneous chemical ignition delay time is much shorter than the fluid dynamic mixing time, which is much shorter than the overall period of the pulse combustor $\left(\tau_{\text {kinetic }} \ll \tau_{\text {mixing }} \ll \tau_{\text {resonant }}\right.$ ).

The initial conditions for the calculation are determined by specifying the fuel/air equivalence ratio and a mixing fraction $f$, which is defined as the mass fraction of fresh fuel and air in the mixture of fuel, air, and combustion products from previous cycles. The fraction of residual combustion products is thus $1-f$.

With this mixing fraction given, the initial mixture temperature $T_{\operatorname{mix}}$ is computed on the basis of energy conservation, since the reactive mixture consists of a fraction $f$ of fuel and air at room temperature $T_{\mathrm{r}}$ and a fraction $1-f$ of residual products assumed to be at their adiabatic flame temperature $T_{\mathrm{ad}}$. Thus, as the mixing fraction increases, $T_{\mathrm{mix}}$ decreases and the ignition delay time increases, since the chemical reaction processes during the delay period have rates that are exponential functions of $T_{\text {mix }}$.

Typical results are illustrated in Fig. 1 for $f=0.3$. The fuel mole fraction $Y_{\text {fuel }}$, the temperature of the mixture, and the energy release rate are plotted as functions of elapsed time. In this example with $\mathrm{CH}_{4}$ as the fuel, the equivalence ratio $\phi$ is 0.8 , and the initial temperature of the mixture $T_{\text {mix }}$ is $1530 \mathrm{~K}$. At the end of the ignition delay period of $1.3 \mathrm{~ms}$, the fuel concentration essentially vanishes. The disappearance of the fuel permits the levels of the important chaincarrying radical species, especially $\mathrm{H}$ and $\mathrm{OH}$, to increase rapidly [14], and subsequent conversion of $\mathrm{CO}$ and $\mathrm{H}_{2}$ to $\mathrm{CO}_{2}$ and $\mathrm{H}_{2} \mathrm{O}$ then results in 

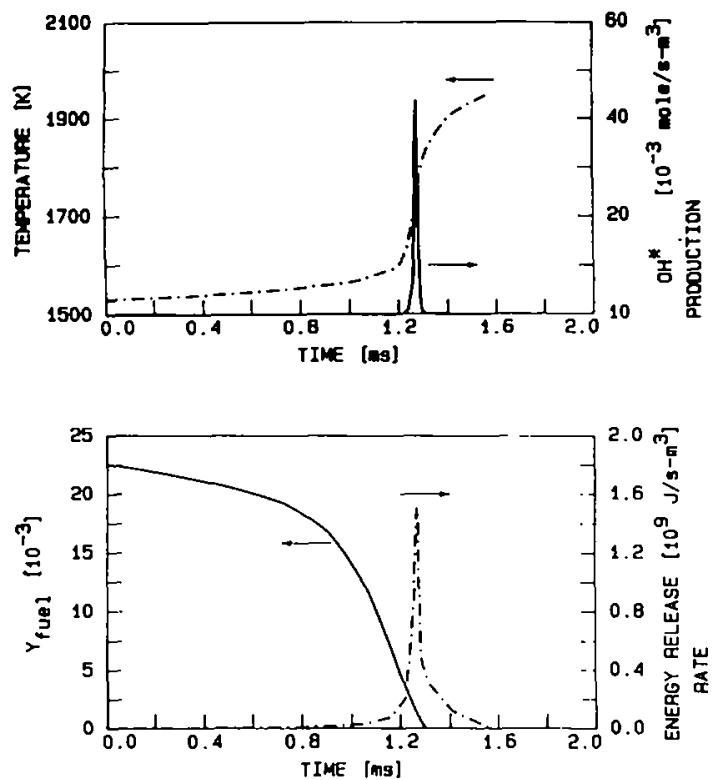

Fig. 1. Typical chemical kinetic calculations showing temperature rise, the production of $\mathrm{OH}^{*}$, the disappearance of fuel, and the evolution of energy release rate.

a rapid temperature increase, as seen in Fig. 1. Also shown in Fig. 1 is the computed production of $\mathrm{OH}^{*}$ resulting from

$\mathrm{CH}+\mathrm{O}_{2} \rightarrow \mathrm{CO}+\mathrm{OH}^{*}$,

which is the main process producing excited $\mathrm{OH}^{*}$ radicals $[15,16]$. The time scale for $\mathrm{OH}^{*}$ emission is governed by the rate of radiative decay of $\mathrm{OH}^{*}$ to the ground state; this characteristic time is approximately $1 \mu \mathrm{s}$ [15]. From these calculations, it is seen that the $\mathrm{OH}^{*}$ emission correlates closely with the rapid energy release phase of the combustion cycle and signals the end of the induction period. For this study, measurements of $\mathrm{OH}^{*}$ chemiluminescence are used as a measure of the energy release rate and as a measure of the ignition delay time.

Keller and Westbrook were guided by this model in their study of the influence of fuel composition on the behavior of pulse combustion [17]. They found that when the homogeneous chemical kinetic ignition delay time was shortened by 0.4 $\mathrm{ms}$, the pulse combustor became "detuned," suggesting that if the chemical kinetic ignition delay time were longer the pulse combustor would operate more stably. Westbrook and Keller [18] applied this model to the search for a set of reactants that would modify the ignition delay time. They found that the addition of small amounts of $\mathrm{N}_{2}$ or $\mathrm{CO}_{2}$ would significantly lengthen $\tau_{\text {kinetic }}$. Since $\mathrm{N}_{2}$ is already the major constituent of air, small amounts of $\mathrm{N}_{2}$ will cause negligible changes in the physical properties of the reactants; therefore only $\tau_{\text {kinetic }}$ (and therefore the total cycle time $\tau_{\text {total }}$ ) will be altered. Following the work of Keller et al. [2], $\mathrm{N}_{2}$ was chosen as a diluent to control $\tau_{\text {kinetic }}$ for this study.

In the present work, the same kinetic model has been used to investigate the effect of homogeneous chemical ignition delay time ( $\left.\tau_{\text {kinetic }}\right)$ on the magnitude of the total ignition delay time. In the laboratory combustor the quantity $f$ is not a well-defined physical variable, since the intake of fresh fuel and air and the mixing with residual products is a very complex and poorly understood process. In performing the homogeneous chemical ignition delay time calculations, the quantity $f$ was chosen so that the variability in $\tau_{\text {kinetic }}$ agreed well with measured values, as will be discussed later. This value of $f$ was then used as a reference case from which $f$ for each operating condition was estimated, based on the mass flow rate and on the frequency of operation, as described in Eq. 1.

$f=f_{\mathrm{r}} \frac{M}{M_{\mathrm{r}}}\left(\frac{\tau}{\tau_{\mathrm{r}}}\right)$

Here $f_{\mathrm{r}}, \tau_{\mathrm{r}}$, and $M_{\mathrm{r}}$ represent the mixing fraction, the characteristic cycle time, and mass flux, respectively, at the reference condition, and $f, \tau$, and $M$ represent the mixing fraction, the characteristic cycle time, and mass flux at the desired condition. In Eq. 1 it is assumed that $f$ should scale linearly with the mass flux, since as the mass flux increases, the fraction of premixed reactants in the combustion chamber also increases. The mixing fraction $f$ should scale linearly with the characteristic cycle time, since the amount of reactants injected with each cycle decreases as the cycle time decreases. Therefore Eq. 1 represents a first-order correction to the value of $f$ for changes in the mass loading and characteristic cycle time. 


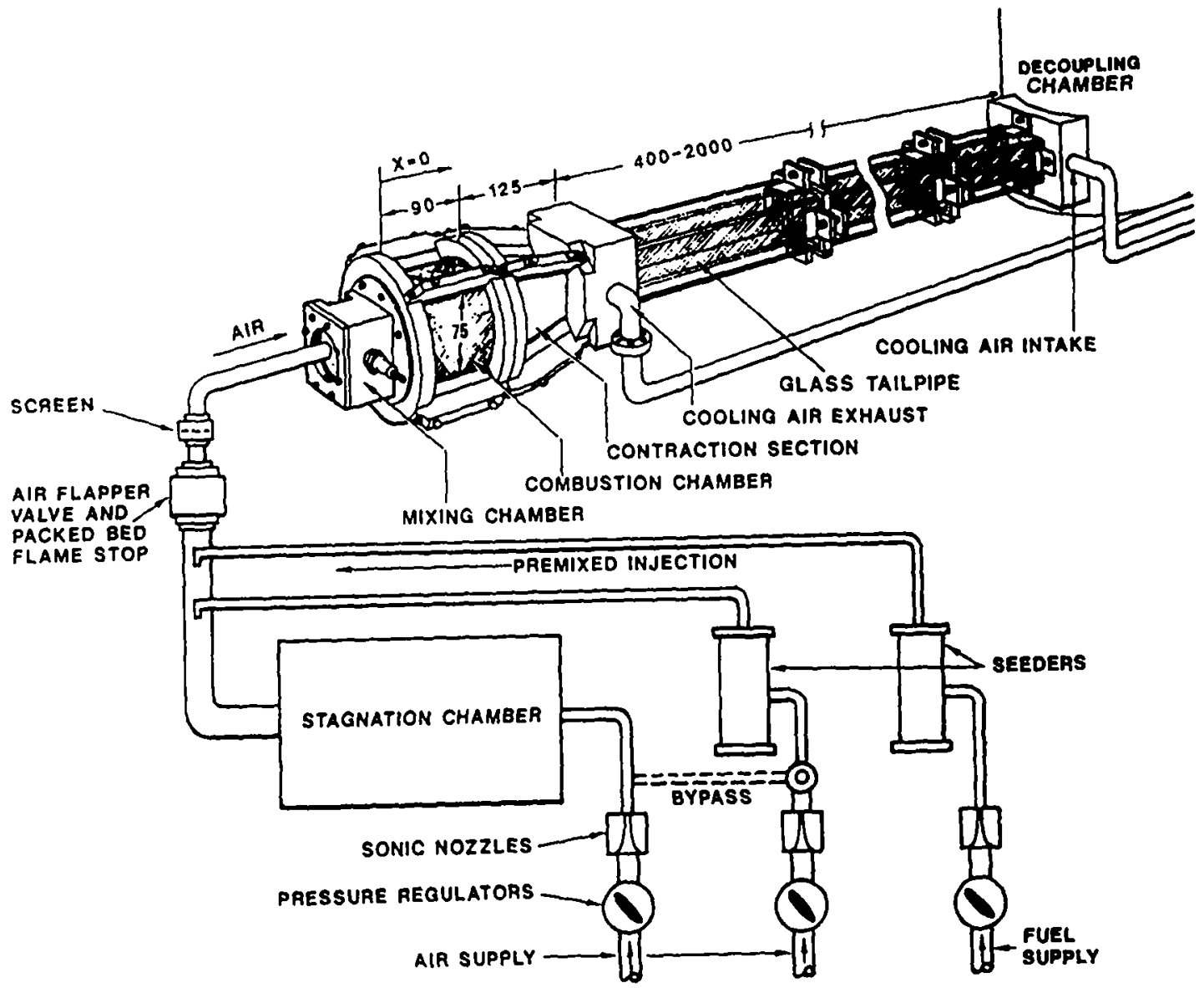

Fig. 2. Schematic of the valved pulse combustor. Dimensions are shown in millimeters.

\section{Experimental Facility}

Figure 2 presents a schematic of the combustor system used in this study. A one-way valve (flapper valve) is located in the reactant supply line just upstream of the axisymmetric mixing chamber. This valve opens and closes depending on the pressure difference between the supply line and the chamber. In this work the combustor was operated in a premixed mode where the fuel and air were mixed upstream of the flapper valve. The injection system for this work was made so that the reactants were injected into the combustion chamber on axis is shown in Fig. 2. The reactants were introduced into the mixing chamber through an orifice plate with seven orifices creating seven small jets.
The mean mass flow rates of the reactants were controlled and metered by the use of sonic nozzles. These flow rates are reported in terms of a mass flux that is calculated by dividing the measured mean mass flow rates by the cross-sectional area of the combustion chamber.

The reactants flow from the mixing chamber into a square-cross-section combustion chamber $75 \mathrm{~mm}$ on a side and $90 \mathrm{~mm}$ in length. A square contraction section follows the combustion chamber and joins the combustion chamber to the tailpipe. The tailpipe is made of a variable number of $200 \mathrm{~mm}$ sections, allowing the total length of the tailpipe to be altered. The tailpipe length for this work was chosen so that the domain of obtainable total ignition delay times was centered about the delay time corresponding to maximum reso- 
nance (see Keller et al. [2]); this criterion yielded a tailpipe length of approximately $600 \mathrm{~mm}$.

\section{Diagnostics}

Measured ignition delay times were obtained through measurements of $\mathrm{OH}^{*}$, which, as noted above, is a measure of energy release rates. These measurements were obtained by placing a photomultiplier tube equipped with a $310 \pm 10 \mathrm{~nm}$ filter at the focal point of a collection lens positioned to collect parallel light generated at $90^{\circ}$ to the streamwise axis of combustion chamber. This position, viewed normal to the axis of the combustor, provided a positionally unbiased, spatially integrated measurement of the amount of $\mathrm{OH}^{*}$ produced in the combustion chamber. In addition to measurements of $\mathrm{OH}^{*}$ chemiluminescence, measurements of characteristic cycle time and combustion chamber pressure were also obtained. The combustion chamber pressure was measured by a pressure transducer with a natural resonance frequency sufficiently fast to resolve the pulse combustor frequency. The pulse combustor characteristic cycle time was deduced from this measurement of pressure.

The total ignition delay time was inferred from ensemble-averaged measurements of $\mathrm{OH}^{*}$ chemiluminescence (denoted by $\left\langle\mathrm{OH}^{*}\right\rangle$ ). These ensemble-averaged data are time-resolved with respect to the combustor cycle by phase-locking on the combustion chamber pressure. The reference time 0 was chosen to be the downward-going zero crossing of the oscillating component of the combustion chamber pressure. All of the ensemble averages were performed over a minimum of 1500 cycles, sufficient to minimize statistical uncertainty. Data at 20 discrete points throughout the combustor's cycle were obtained for the ensemble averages. Figure 3 shows a typical $\left\langle\mathrm{OH}^{*}\right\rangle$ curve plotted against cycle time normalized by the characteristic cycle time. Shown also is a typical combustion chamber pressure curve. The vertical arrow indicates the total ignition delay time defined as the first point of inflection in the $\left\langle\mathrm{OH}^{*}\right\rangle$ chemiluminescence data $\left(\partial^{2}\left\langle\mathrm{OH}^{*}\right\rangle / \partial t^{2}=0\right)$.

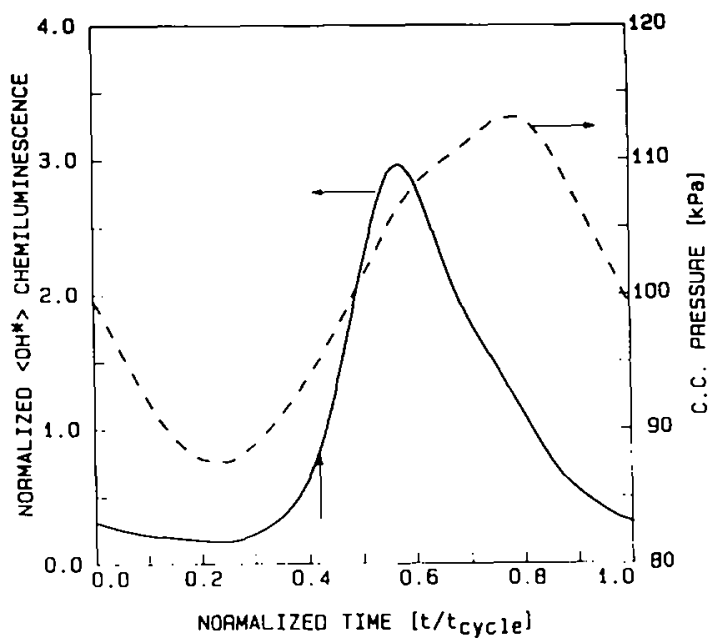

Fig. 3. Typical normalized $\left\langle\mathrm{OH}^{*}\right\rangle$ chemiluminescence and combustion chamber pressure versus normalized cycle time. The vertical arrow indicates the end of the induction period as defined to be the first inflection point in the $\left\langle\mathrm{OH}^{*}\right\rangle$ chemiluminescence curve.

\section{EXPERIMENTAL INVESTIGATION}

\section{Quantification of Characteristic Times Model Calibration}

The total ignition delay time was determined for two independent studies; in the first the characteristic mixing time ( $\left.\tau_{\text {mixing }}\right)$ was varied, and in the second the homogeneous chemical kinetic ignition delay time $\left(\tau_{\text {kinetic }}\right)$ was varied. Recall that Keller et al. [2] established that the influence of these two characteristic times on the total ignition delay time is, at least to first order, independent of the other characteristic times in the system. From these studies information was obtained to calibrate the mixing model under conditions of constant chemical kinetic time and to calibrate the kinetics model under conditions of constant mixing times.

The physical and chemical processes leading to ignition are highly coupled, temporally and spatially varying processes that involve turbulent mixing, injection rates, chemical kinetics, and so on. Nevertheless, although it is recognized that these important processes overlap to some extent in a nonlinear fashion, a significant amount of useful information can be extracted from the experimen- 
tal results by treating the influence of processes as being independent. Mathematically, this type of analysis should be appropriate as long as the operating parameters are not changed too drastically. For example, this type of analysis should not be expected to predict correctly the results of large simultaneous variations in both mixing rates and kinetic time scales.

The functional form that combines these processes into a total ignition delay is not known. Following Spalding's eddy breakup model [19], where the turbulent burning rates are modeled as the sum of the characteristic times for turbulent mixing and chemical reactions, $\tau_{\text {total }}$ was calculated as the sum of $\tau_{\text {mixing }}$ calculated from the jet mixing model and $\tau_{\text {kinetic }}$ calculated from the homogeneous chemical kinetics model. This provides a worst case because the influence of both the mixing and chemical kinetics times on the total ignition delay time will be overestimated since these processes, in reality, occur simultaneously. The conditions chosen for the calibration of the mixing model [a mass flux of 1.0 $\mathrm{kg} /\left(\mathrm{s} \cdot \mathrm{m}^{2}\right)(4.549 / \mathrm{s})$, an equivalence ratio of 1.0 , and a diluent mass fraction of 0.1$]$ were such that the magnitude of the chemical kinetic ignition delay time, calculated as discussed below, would be small ( $\tau_{\text {kinetic }} \approx 0.37 \mathrm{~ms}$ ). Using this approach, the adjustable parameter $C_{\text {pent }}$ in the mixing model was fixed so that the calculated $\tau_{\text {total }}$ approximately equalled the measured $\tau_{\text {total }}$. A value of $C_{\text {pent }}=0.21$ was found. This value is consistent with the improved mixing characteristics of pulsating turbulent jets $[7,9]$ and serves as the mixing model calibration used throughout the rest of the study.

Shown in Fig. 4 are the data used to calibrate the chemical kinetics model. Plotted are the characteristic ignition delay times as calculated by the chemical kinetics model and as measured from the experiment for a wide range of diluent mass fractions and constant mixing times; hence the changes in the measured total ignition delay time are due only to the changes in the homogeneous chemical kinetic ignition delay time. All the times presented in Fig. 4 are time differences (the ignition delay time minus the ignition delay time at the low-

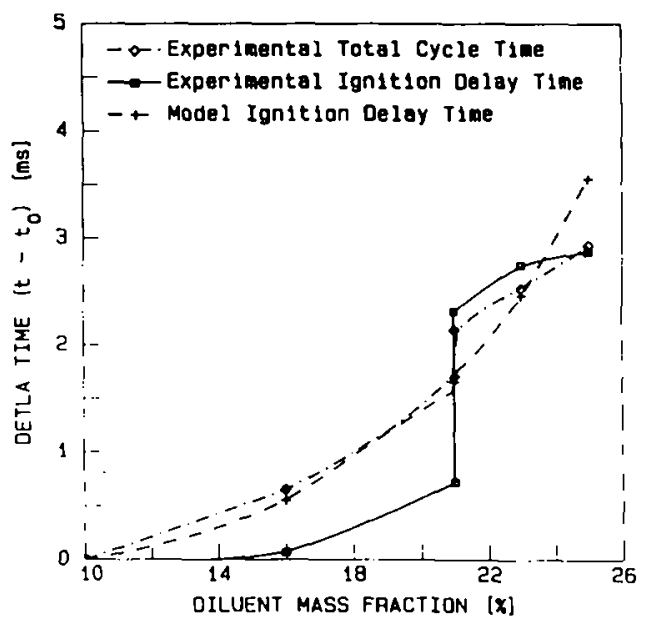

Fig. 4. Comparison between the difference in ignition delay times $\left(\tau-\tau_{0}\right)$ for varying amounts of diluent and constant mass flux $\left[1.0 \mathrm{~kg} /\left(\mathrm{s} \cdot \mathrm{m}^{2}\right)\right]$. The mixture fraction $f$ used in the chemical kinetics model was chosen so that the largest measured $d$ ference in total ignition delay time matched the calculated values

est diluent condition, $\tau-\tau_{0}$ ). The largest overall time difference was used to fix the mixing fraction $f$. Calibrating the chemical kinetics model in this way, $f=0.24$. It should be noted that the homogeneous chemical kinetics ignition delay time is an exponential function of $f$, through the initial temperature, as discussed above and hence the agreement between the measured and the calculated ignition delay times shown in Fig. 4 is considered excellent. The discontinuity seen in the experimental data in Fig. 4 will be discussed below.

\section{Fluid Dynamic Mixing Times}

The calculated mixing rates were compared with experimentally measured energy release rates. These two sets of curves are compared in Fig. 5 for three values of the inlet mean mass flux. The solid lines represent $\left\langle\mathrm{OH}^{*}\right\rangle$, and the dashed lines represent the model calculations. These data were normalized so that the areas under the curve are, by definition, equal to unity. The conditions shown in Fig. 5 (equivalence ratio $\phi=1.0$ and diluent fraction $\psi=0.1$ ) were selected so that the homogeneous chemical ignition delay time could be assumed constant and small, making the measured $\left\langle\mathrm{OH}^{*}\right\rangle$ chemiluminescence a good approximation 


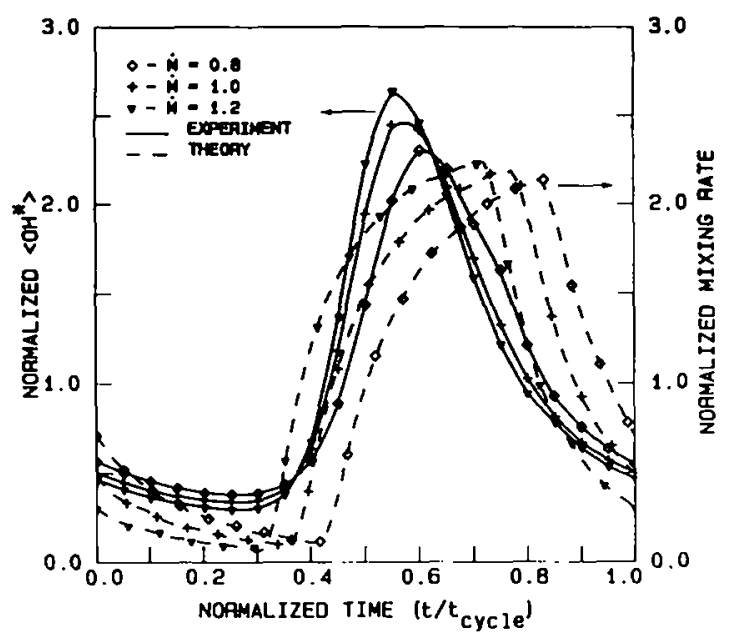

Fig. 5. Comparison between the measured normalized $\left\langle\mathrm{OH}^{*}\right\rangle$ and the calculated fluid dynamic mixing rate for three different mass flux cases and constant $N_{2}$ diluent $(\psi=0.10)$ with different characteristic mixing times ( $\left.\tau_{\text {mixing }}\right)$.

to the mixing rates in the combustor. In the mixing model, the mixing-dominated ignition delay time is defined in a fashion similar to that above in that it is the first point of inflection in the calculated mixing rate data. As shown in Fig. 5, the predicted values of mixing rates are in good agreement with the experimentally observed values. The model correctly predicts the onset of reaction and the end of the induction period. As the mass flux increases, $\tau_{\text {total }}$ decreases. Close examination of Fig. 5 shows that the model predicts a stronger trend in the ignition delay time with mass flux than the experimental results show. The mixing model assumes a constant homogeneous chemical kinetic delay time; although care was taken to hold $\tau_{\text {kinetic }}$ constant, it is indeed a function of mass flux. In fact, according to Eq. $1, f$ increases as the mass flux is increased and thus $\tau_{\text {kinetic }}$ increases. Hence, the measured results will not show as strong a trend with mass flux due to the competing effects with $\tau_{\text {kinetic }}$.

Although the onset of reaction is predicted by the model, the computed curves are substantially broader than the experimental observations, indicating a longer duration for the energy release phase. It was not possible to reproduce the model conditions exactly with the experiment. The experimental configuration used in conjunction with this model actually consists of an array of jets that inject reactants parallel to the axis of the pulse combustion chamber. In performing the mixing calculations, the possible interaction of these jets was neglected. This weakness in the correspondence between experimental and modeled configurations may be the cause of the model's underprediction of the burning rates.

\section{Chemical Ignition Delay Times}

A comparison between changes in the measured total ignition delay time $\tau_{\text {total }}$ and the calculated homogeneous ignition delay time $\tau_{\text {kinetic, calc }}$ was also made. In the reference case the instantaneous energy release rate occurs early in the cycle, prior to the peak in the pressure root mean square about the mean, $\boldsymbol{P}_{\text {rms }}$ (see Keller et al. [2]). The kinetic ignition delay time is then retarded by adding $\mathrm{N}_{2}$ while maintaining constant mixing characteristics. The results are shown in Fig. 6. These data are for conditions of varying $\mathrm{N}_{2}$ concentration and constant mass flux and equivalence ratio $\phi$ at $1.0 \mathrm{~kg} /\left(\mathrm{s} \cdot \mathrm{m}^{2}\right)$ and 1.0 , respectively. Two overall
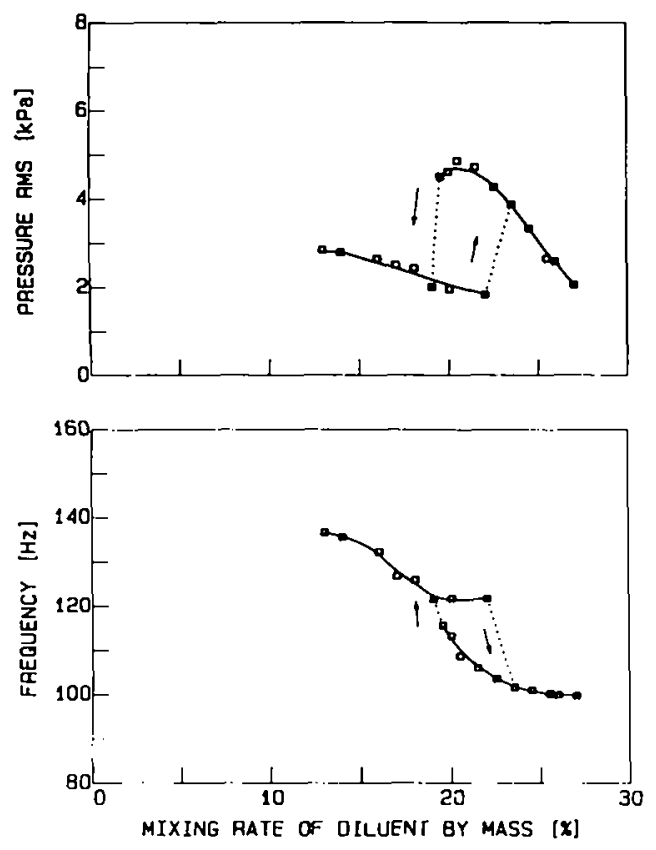

Fig. 6. Pressure $P_{\mathrm{rms}}$ and frequency shown as a function of the fraction of $N_{2}$ diluent. These data are for a mass flux of $1.0 \mathrm{~kg} /\left(\mathrm{s} \cdot \mathrm{m}^{2}\right)$ and an equivalence ratio of 1.0 . 
trends are observed. Starting with low amounts of $\mathrm{N}_{2}$, as the concentration of $\mathrm{N}_{2}$ is increased, decreasing the amount of fuel, both $P_{\text {rms }}$ and the frequency decrease. The decrease in $P_{\text {rms }}$ occurs because less energy is being released per cycle (due to dilution), and the frequency decreases because the longer kinetic times lead to energy release later and later in the cycle.

This trend continues until the diluent fraction is approximately 0.2 , where an instability occurs. At this point it was observed that the pressure $P_{\text {ms }}$ suddenly increased by approximately a factor of 2 and the operating frequency dropped significantly. As the amount of dilution continued to increase, the pressure maximum and frequency continued to decrease. In the instability region an interesting hysteresis phenomenon was found. It is possible that the instability region and hysteresis are a result of changes in the shape of the instantaneous energy release rate. This is one of the subjects of our current research.

Measurements of $\left\langle\mathrm{OH}^{*}\right\rangle$ for six cases with different homogeneous ignition delay times are presented in Fig. 7. Normalized $\left\langle\mathrm{OH}^{*}\right\rangle$ chemiluminescence is plotted as a function of normalized cycle time. As shown in the figure, as the amount of diluent is increased, the energy release rate

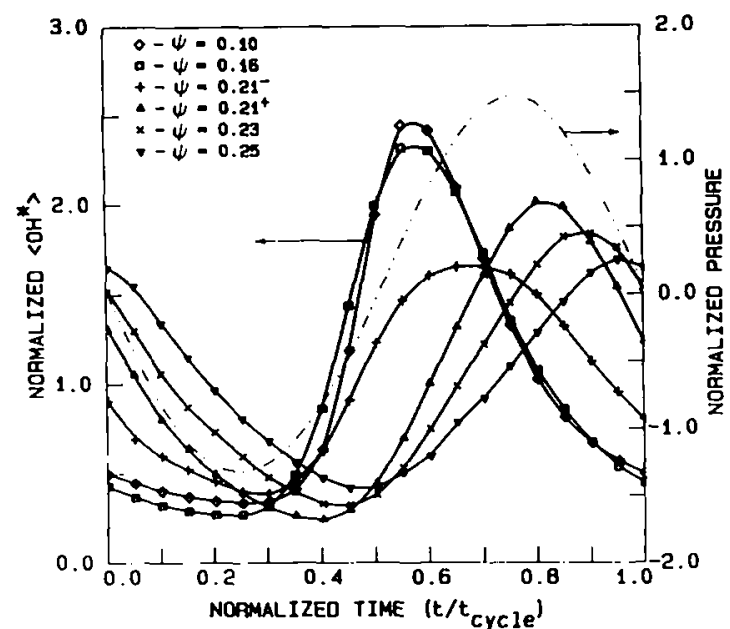

Fig. 7. Shown is $\left\langle\mathrm{OH}^{*}\right\rangle$ chemiluminescence as a function of normalized cycle time. Six cases of different $\mathrm{N}_{2}$ diluent mass fractions $(\psi)$, and hence different homogeneous chemical kinetic ignition delay times $\left(\tau_{\text {kinetic }}\right)$ are presented. These data were taken at a constant mass flux of $1.0 \mathrm{~kg} /\left(\mathrm{s} \cdot \mathrm{m}^{2}\right)$.

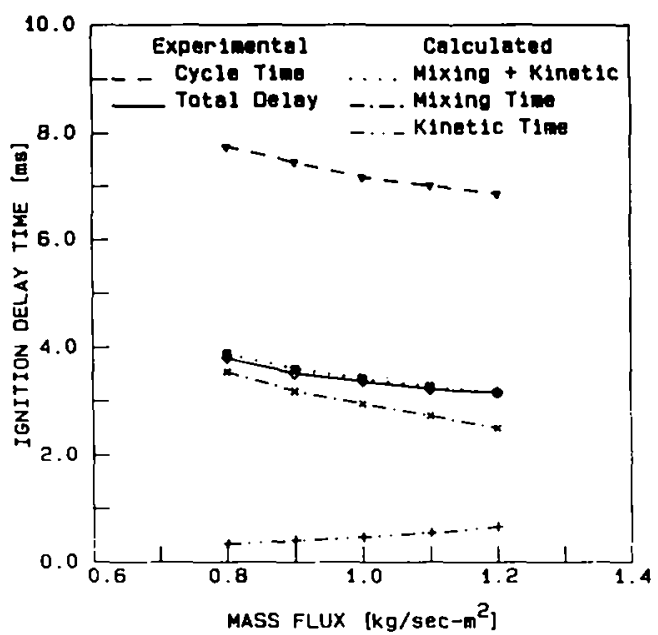

Fig. 8. Characteristic combustor times (normalized by the cycle time) as a function of mass flux and constant $\mathrm{N}_{2}$ diluent mass fraction of $\psi=0.10$. Presented are the calculated homogeneous chemical kinetic ignition delay time, the calculated characteristic mixing time, the measured total ignition delay time, and the calculated total ignition delay time using the linear assumption.

(depicted by the $\left\langle\mathrm{OH}^{*}\right\rangle$ chemiluminescence data) shifts later and later in the cycle. This is due to the fact that the homogeneous chemical kinetic ignition delay time is getting longer and longer. Also, as the amount of $\mathrm{N}_{2}$ is increased, the width of the $\left\langle\mathrm{OH}^{*}\right\rangle$ chemiluminescence curve increases. This effect is also due to the slower chemistry. The case with an $\mathrm{N}_{2}$ diluent fraction of 0.21 is repeated twice, showing the instantaneous energy release rate on the low $\left(0.21^{-}\right)$and high $\left(0.21^{+}\right)$sides of the instability shown in Fig. 6. The shape of the $\left\langle\mathrm{OH}^{*}\right\rangle$ curve for $0.21^{+}$is more peaked than the sinusoidal shape of the $0.21^{-}$case, indicating that the shape for maximum resonant pressure wave reinforcement is more peaked than sinusoidal.

\section{Total Ignition Delay Time}

The ability of these models to accurately predict the total ignition delay time was also examined. Figure 8 is a comparison of the calculated results from the fluid dynamic mixing, the chemical kinetic models, the calculated total ignition delay time, and the measured ignition delay time. The behavior of both the calculated mixing time and the calculated homogeneous chemical kinetic time 
with increasing mass flux rates is shown in the figure; the mixing model predicts that as the mass flux increases, the mixing time decreases, while the chemical kinetics model predicts the opposite trend for the kinetic time.

The relative importance of the fluid dynamic mixing time and the homogeneous chemical kinetic time can be seen in Fig. 8. The calculated homogeneous chemical kinetic delay time ranges from $5 \%$ to approximately $12 \%$ of the total cycle time, whereas the calculated mixing times range from $45 \%$ to $35 \%$ of the total cycle time. The measured total delay time is almost constant at approximately $50 \%$ of the total. From these results it is seen that the fluid dynamic mixing time is on the order of $90 \%$ of the total ignition delay time and the homogeneous chemical kinetic ignition delay time makes up the remaining fraction. Since the chemical kinetics time represents a small fraction of the total ignition delay time, the error introduced by a linear assumption will not be large, and an estimate of the total ignition delay time can be made by simple addition of the characteristic mixing time and the homogeneous chemical kinetic ignition delay time. This calculation is also shown in Fig. 8. As a first-order approximation this linear assumption produces results in good agreement with the experimental observations.

\section{SUMMARY}

A systematic study of the quantification of characteristic times that contribute to the total ignition delay time in a pulse combustor has been performed. The total ignition delay time was measured and calculated for a wide range of operating conditions.

The fluid dynamic mixing time was measured by operating the combustor at conditions where the homogeneous chemical kinetic ignition delay time was constant and negligible with respect to the fluid dynamic mixing time. This measurement was used to fix the one adjustable parameter in the mixing model. The trends predicted by this model for these conditions closely resembled the trends found in the experiment. The influence of the homogeneous chemical kinetic ignition delay time was measured by maintaining the fluid dynamic mixing time constant and varying the homogeneous chemical kinetic ignition delay time over a wide range. These measurements were used to fix the one adjustable parameter in the kinetics model.

Guided by previous work, which established that the characteristic times of interest in the pulse combustor contribute nearly independently to the total ignition delay time, a simple yet powerful hypothesis was developed. Not only are these times independent in their contributions to the total ignition delay time, but they can be added together linearly to obtain the total ignition delay time. This concept was successfully tested by direct experimental verification at conditions of varying homogeneous chemical kinetic ignition delay time and varying fluid dynamic mixing time. The measured total ignition delay time and the sum of the calculated ignition delay times were found to have the same trend and shape and comparable values. Thus, the models, although simplifications of a very complex problem, have potential utility in the design and understanding of pulse combustion systems.

We wish to express our gratitude to Ichiro Hongo of Toshiba Corporation for assistance in the laboratory during the long hours of data acquisition. This work was supported by the U.S. Department of Energy, Office of Energy Utilization Research, Division of Energy Conversion and Utilization Technologies and the Gas Research Institute.

\section{REFERENCES}

1. Kardos, P., personal communication, fall 1985.

2. Keller, J. O., Dec, J. E., Westbrook, C. K., and Bramlette. T. T., Pulse Combustion: The Importance of Characteristic Times, Combust. Flame 75:33 (1987).

3. Rife, J. M., and Heywood, J. B., Photographic and Analytical Study of Diesel Combustion Machine, $S A E$ Thans. 83:2942-2961 (1974).

4. Bramlette, T. T., The Role of Fluid Dynamic Mixing in Pulse Combustors, Sandia National Laboratories Report SAND87-8622, 1987.

5. Abramovich, G. N., The Theory of Turbulent Jets, MIT Press, Cambridge, Mass., 1963. 
6. Breidenthal, R. E. The Turbulent Exponential Jet, Phys. Fluids 29:2346-2347 (1986).

7. Kato, S. M., Groenemegen, B. C., and Breidenthal, R. E., Turbulent Mixing in Nonsteady Jets, $A I A A J$. 25:165-168 (1987).

8. Keller, J. O., and Saito, K., Measurements of the Combusting Flow in a Pulse Combustor, Combust. Sci. Technol. 53:2-3 (1987).

9. Binder, G., and Favre-Marinet, M., Mixing Improvements in Pulsating Turbulent Jets, in Proc. ASME Symposium on Fluid Mechanics of Mixing (E.M. Uram, Ed.), pp. 167-172, Atlanta, Ga, June 1973.

10. Lund, C. M., HCT-A General Computer Program for Calculating Time Dependent Phenomena Involving OneDimensional Hydrodynamics, Transport, and Detailed Chemical Kinetics, University of California Lawrence Livermore Laboratory report UCRL-52504, 1978.

11. Westbrook, C. K., An Analytical Study of the Shock Tube Ignition of Mixtures of Methane and Ethane, Combust. Sci. Technol. 20:5 (1979).

12. Westbrook, C. K., and Pitz, W. J., A Comprehensive Chemical Kinetic Reaction Mechanism for Oxidation and Pyrolysis of Propane and Propene, Combust. Sci. Technol. 37:117 (1984).

13. Pitz, W. J., Westbrook, C. K., Proscia, W. M., and Dryer, F. L., A Comprehensive Chemical Kinetic Reaction Mechanism for the Oxidation of $n$-Butane, Twenti- eth Symposium (International) on Combustion. The Combustion Institute, Pittsburgh, Pa., 1985, p. 831.

14. Westbrook, C. K., and Dryer, F. L., Chemical Kinetic Modeling of Hydrocarbon Combustion, Prog. Energy Combust. Sci. 10:1-57 (1984).

15. Gaydon, A. G., The Spectroscopy of Flames, Chapman and Hall, London. 1974.

16. Crosley, D. R., and Dyer, M. J., Two-Dimensional Imaging of Laser-Induced Fluorescence in $\mathrm{OH}$ in a Flame, Proc. International Conference of Lasers '82, Dec. 13-17, 1982.

17. Keller, J. O. and Westbrook, C. K., Response of a Pulse Combustor to Changes in Fuel Composition, Twenty-First Symposium (International) on Combustion, The Combustion Institute, Pittsburgh. Pa.. 1987. pp. 547-555.

18. Westbrook, C. K., and Keller, J. O., Chemical Kinetic Analysis of Pulse Combustors, presented at the 1986 International Gas Research Conference. Toronto, Canada, Sept. 8-11, 1986.

19. Spalding, D. B., Mixing and Chemical Reaction in Steady Confined Turbulent Flames, Thirteenth Symposium (International) on Combustion. The Combustion Institute, Pittsburgh, Pa., 1971, pp. 649-657.

Received 4 April 1988; revised 2 December 1988 